\title{
Rare diseases
}

Keywords: medicinal chemistry $\bullet$ rare disease $\bullet$ therapeutic modality

This special issue highlights the application of medicinal chemistry to the pursuit of transformative medicines for orphan and genetic diseases, which affect millions of individuals worldwide, and for which there is a huge unmet medical need. Many of the current themes of rare disease research are explored, including the necessary diversification of medicinal chemistry in the field. The impressive breadth of therapeutic modalities explored in the issue illustrates the various innovative approaches being applied by medicinal chemists and biologists in the fight against rare diseases, which include pharmacological chaperones, multitarget drugs, theranostics, proteostatic modulators, protein replacement, gene therapy, RNAi, antisense, monoclonal antibodies (mAbs), polymerization blockade, stem cell therapy and transcriptional and epigenetic modulators. The diseases covered are equally diverse, and include $\alpha 1$-antitrypsin deficiency, galactosemia, prion diseases, cystic fibrosis, lysosomal storage diseases (e.g., Gaucher's disease, Pompe's disease and mucopolysaccharidoses), spinal muscular atrophy, sickle cell disease and rare channelopathies.

\section{Beyond the traditional confines of medicinal chemistry}

The role of the medicinal chemist in this challenging new era is changing since therapeutically relevant chemical space and novel pharmacological opportunities that rely on molecular design go well beyond traditional boundaries [1,2]. In this new paradigm, several groups have challenged the standard process of small-molecule drug discovery to ensure medicinal chemistry is primed to deliver the greatest impact to drug research, agnostic to the therapeutic modality. A significant opportunity for medicinal chemistry in rare disease research is the successful application of chemistry at the interface with biology. In our experience, chemical biology should be 'technology-agnostic' - the initial focus is in defining the right question, and then using the right technology to answer that question. As an example, the advances in next-generation sequencing can help define the exact molecular cause of a rare genetic disease [3], which greatly facilitates the creation of a relevant screen sequence to deliver molecules possessing the desired efficacy in the clinic, so addressing the major cause of drug attrition [4]. However, phenotypic and pathway screening in this way unearths molecules with unknown molecular modes of action, and therefore a focus area for chemical biology will be target-mechanism identification and validation of that target to accelerate drug discovery and development. There are some examples reviewed in the issue, and I expect there to be many more in the years to come. Rare disease chemical biology will therefore unearth even more therapeutic opportunities in the future and hopefully increase the rate at which new medicines are discovered (Figure 1).

\section{Evolving collaborative partnerships}

Another important theme that pervades debate in this area, and that is evident in this issue, is that of collaboration, often between

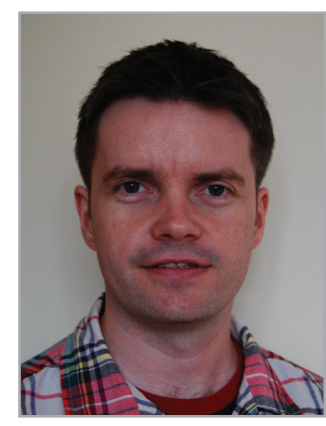

Lyn H Jones

Rare Disease Chemistry, Worldwide Medicinal Chemistry, Pfizer, 610 Main Street, Cambridge, MA 02139, USA lyn.jones@pfizer.com 


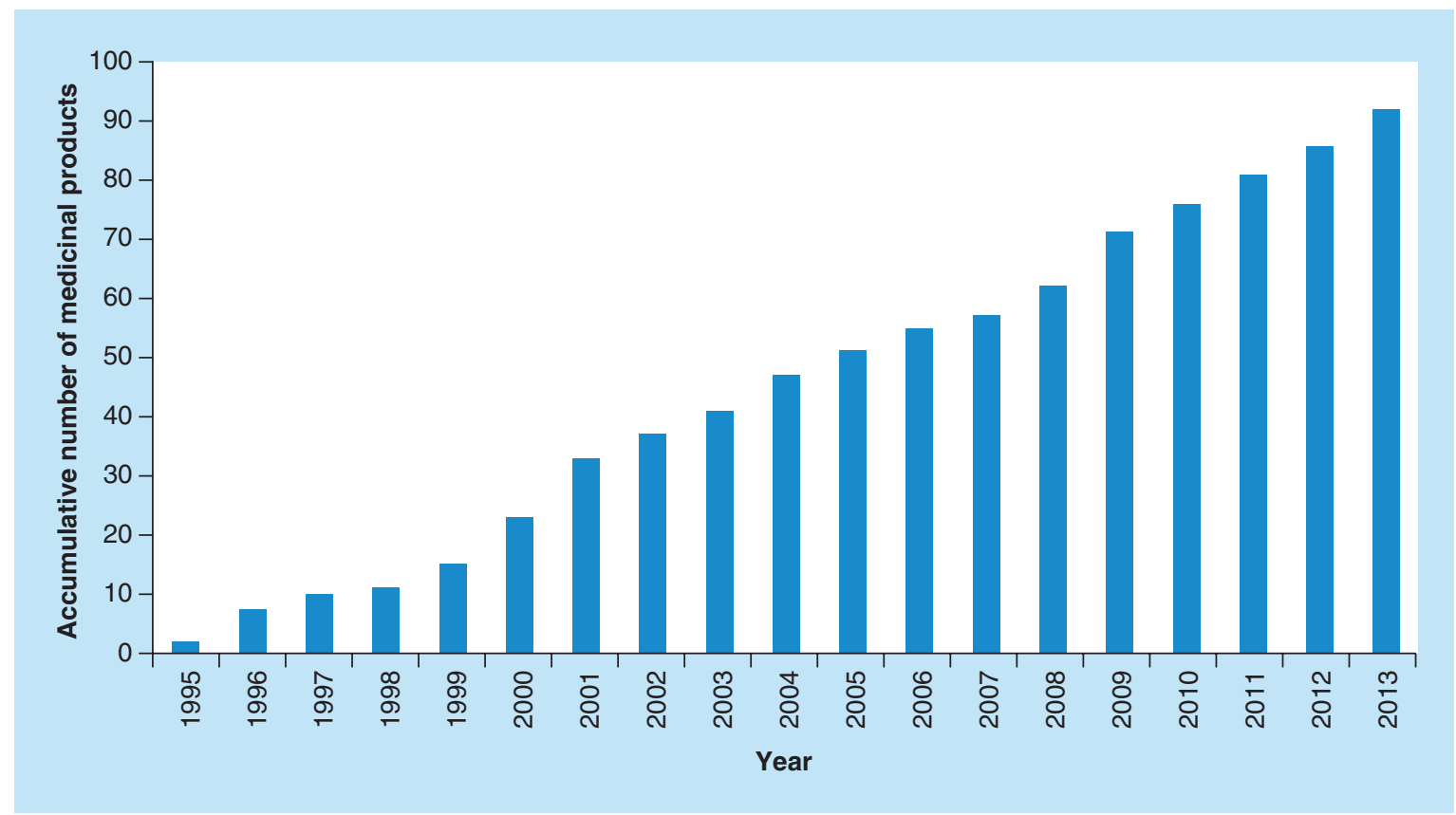

Figure 1. Number of rare disease medicinal products in Europe (data taken from January 2013 Orphanet Report Series: list of medicinal products for rare diseases in Europe).

pharmaceutical/biotechnology companies and academic institutions. These partnerships often boost innovation, enhance knowledge spillovers and can lead to significant advances in $\mathrm{R} \& \mathrm{D}[5]$. The recent movements within venture philanthropy and family offices are also noteworthy $[6,7]$. Equally important are the strong links between $\mathrm{R} \& \mathrm{D}$ and patient advocacy groups that are essential to facilitate drug discovery in these areas.

This is a very exciting time for rare disease research and I am very thankful to the authors who have shared their expertise and insight in this special issue. It will

\section{References}

1 Jones LH, McKnight AJ. Biotherapeutics: recent developments using chemical and molecular biology. In: RSC Drug Discovery Series. RSC Publishing, Cambridge, UK (2013).

2 Jones LH. New Frontiers in Chemical Biology. Bunnage ME (Ed.). RSC Publishing, Cambridge, UK (2011).

3 Boycott KM, Vanstone MR, Bulman DE, MacKenzie AE. Rare-disease genetics in the era of next-generation sequencing: discovery to translation. Nat. Rev. Genet. 14, 681 (2013). provide valuable discussion points and will appeal to those working in all areas of rare disease research.

\section{Financial \& competing interests disclosure}

LH Jones is an employee and shareholder of Pfizer. The author has no other relevant affiliations or financial involvement with any organization or entity with a financial interest in or financial conflict with the subject matter or materials discussed in the manuscript apart from those disclosed.

No writing assistance was utilized in the production of this manuscript.

4 Bunnage ME. Getting pharmaceutical R\&D back on target. Nat. Chem. Biol. 7, 335 (2011).

5 Wright BD, Drivas K, Lei, Z, Merrill SA. Industry-funded academic inventions boost innovation. Nature 507, 297 (2014).

6 Jarvis LM. Advancing R\&D by venture philanthropy. Chem. Eng. News 90, 14 (2012).

7 Senior M. Family offices bolster early-stage financing. Nat. Biotechnol. 31, 473 (2013). 